\title{
THE CONSTANTS IN WARING'S PROBLEM FOR ODD POWERS
}

BY R. D. JAMES

1. Introduction. In a previous paper* the writer determined the constants in the Hardy-Littlewood analysis of Waring's problem. It was then possible to obtain new universal theorems. The proof depended on the following results.

Theorem A. (Theorem 46, page 429, and (C), page 442 in paper T.) Let $k \geqq 6$ be an integer; $s$ an integer $; a=1 / k$;

$$
\begin{aligned}
& d=[\log (k-1) / \log 2] ; \quad D=(d+2)(k-1)-2^{d+1}+0.1 ; \\
& s_{2}=4+\zeta_{k}=4+\left[\frac{(k-2) \log 2-\log k+\log (k-2)}{\log k-\log (k-1)}\right] ; \\
& \eta=\frac{D(s-2)+k 2^{k-2}\left(1+(1-a)^{s_{2}-2}\right)}{(s-2)-(k-2) 2^{k-2}-k} .
\end{aligned}
$$

Then every integer $n>C$, where $\log _{e} C=20 k^{3} 2^{\eta}$, is a sum of $s+s_{2}$ kth powers.

The proof of universal theorems was greatly simplified by Dickson's new method. Using Theorem A and very short tables he proved that $\dagger$ every integer is a sum of 259 seventh powers, 575 eighth powers, 981 ninth powers, 10711 twelf th powers, $\cdots$. All these results are considerably better than those obtained by algebraic methods.

In another paper $\ddagger$ the writer obtained new asymptotic results for odd powers. It is the purpose of the present note to point out that the constants of this paper may also be evaluated by the methods of paper $\mathrm{T}$. This leads to still better universal

* Transactions of this Society, vol. 36 (1934), pp. 395-444. This will be referred to as paper $\mathrm{T}$.

$\dagger$ This Bulletin, vol. 39 (1933), Theorems 15-18, pp. 713-714.

$\ddagger$ Proceedings of the London Mathematical Society, (2), vol. 37 (1934), pp. 257-291. This will be referred to as paper $P$. 
theorems for odd powers. Corresponding to Theorem A we have the following theorem.

ThEOREM B. Let $k \geqq 7$ be an odd integer; $t \leqq k 2^{k-2}$ an integer; $a=1 / k ; b=1 /(k-1)$;

$d_{a}=[\log (k-1) / \log 2] ; \quad d_{b}=[\log (k-2) / \log 2] ;$

$D_{a}=\left(d_{a}+2\right)(k-1)-2^{d_{a}+1}+0.1 ; \quad D_{b}=\left(d_{b}+2\right)(k-2)-2^{d_{b}+1}+0.1 ;$

$s_{2}=4+\zeta_{k}$, where $\zeta_{k}$ was defined in Theorem A;

$$
\eta=\frac{D_{a}(2 k-1)(k-1)+D_{b} k(2 t-4)+2^{k-2} k(k-1)\left(1+(1-a)^{s_{2}-2}\right)}{(2 k-1)(k-1)+k(2 t-4)-2^{k-2} k(k-3)-k(k-1)} ;
$$

$v=4$ when $k$ is divisible by $15, v=2$ in all other cases. Then every integer $n>C^{\prime}$, where $\log _{e} C^{\prime}=25 k^{3} 2^{\eta}$, is a sum of $2 t+s_{2}+2 v$ $+2 k-1 k$ th powers.

The use of this theorem instead of Theorem A enabled Dickson* to prove that every integer is a sum of 4425 eleventh powers. The Hardy-Littlewood result showed only that every sufficiently large integer was a sum of 4687 eleventh powers.

2. Changes Required for $C_{100}$. In order to obtain Theorem B there is one change which must be made in paper $\mathrm{P}$. The constant $C_{99}$ which occurs in the proof of Theorem 3 (page 289) is evaluated in precisely the same way as the constant $C$ in paper $\mathrm{T}$, and we shall not repeat the discussion. However, in Lemma 33 (page 290) the constant $C_{100}$ depends on a prime number theory constant which has not been evaluated. Accordingly we replace Lemma 33 by the following lemma.

Lemma A. Define $k, t$, and $v$ as in Theorem B. Let

$p=3$ when $k$ is prime to 6 ,

$p=5$ when $k$ is divisible by 3 and prime to 10 ,

$p=2$ in all other cases.

Then given any integer $w>p^{(k+6) k^{2}}$ there exist $2 v$ kth powers $w_{1}, w_{2}, \cdots, w_{2 v}$ and integers $\mu \geqq 2$ and $W$ such that

$$
w-2 t p^{(k+2+\mu) k^{2}}-\sum_{\alpha=1}^{2 v} w_{\alpha}^{k}=p^{(k+2+\mu) k^{2}} W,
$$

* Journal of the London Mathematical Society, vol. 9 (1934), pp. 201206. 
where

$$
\left(2^{k}-4\right) p^{(k+2+\mu)\left(k^{2}-k\right)} \leqq W \leqq\left(2^{k}-2\right) p^{(k+2+\mu)\left(k^{2}-k\right)} .
$$

The integer $W$ satisfies the inequalities $(8 \cdot 32)$, for the value $N=p^{(k+2+\mu) k}$, on page 288 of. paper P. For by $(2 \cdot 31)$, page 258 , we have

$$
\begin{aligned}
2 t(P & (M)+1)+N^{k-1} \leqq 2 t(P(B N)+1)+N^{k-1} \\
& =2 t\left\{(1+B)^{k}+(1-B)^{k}-2\right\} N^{k-1}+2 t+N^{k-1} \\
& <\left(2^{k}-6\right) N^{k-1}+2 t+N^{k-1}<\left(2^{k}-4\right) N^{k-1},
\end{aligned}
$$

since we have $2 t \leqq k 2^{k}<2^{2 k}<p^{(k+2+\mu)\left(k^{2}-k\right)}=N^{k-1}$.

3. Evaluation of $C^{\prime}$. We shall now prove Lemma $A$ and then show that the constant $C^{\prime}$ in Theorem $\mathrm{B}$ is equal to

$$
v p^{(k+2) k^{2}}+\left(2 t+2^{k}\right)\left(2^{k}-4\right)^{-1} p^{k^{2}} C_{99}^{k /(k-1)} .
$$

Knowing $\log _{e} C_{99}$ (evaluated by the methods of paper $\mathrm{T}$ ) we can therefore find $\log _{e} C^{\prime}$. The result is $\log _{e} C^{\prime}<25 k^{3} 2^{\eta}$.

To prove Lemma $A$, we require two additional lemmas.

Lemma B. Let $m(\geqq 1)$ and $E$ be integers; $P \geqq 0, Q \geqq 0$, and $P+(m-1)^{k} \leqq E \leqq Q$. Then there exist at least $m$ consecutive integers $i_{\beta} \geqq 0,(\beta=1,2, \cdots, m)$, which satisfy the inequalities

$$
P \leqq E-i_{\beta}^{k} \leqq P+k m Q^{(k-1) / k} \text {. }
$$

Proof. Let $\delta=(E-P)^{1 / k}$. Then $[\delta] \geqq m-1$, and we have

$$
\begin{aligned}
P & =E-\delta^{k} \leqq E-[\delta]^{k} \leqq E-([\delta]-m+1)^{k} \\
& =P+\delta^{k}-([\delta]-m+1)^{k} \leqq P+k(\delta-[\delta]+m-1) \delta^{k-1} \\
& \leqq P+k m \delta^{k-1}=P+k m(E-P)^{(k-1) / k} \\
& \leqq P+k m Q^{(k-1) / k} .
\end{aligned}
$$

The integers $[\delta]-m+1,[\delta]-m+2, \cdots,[\delta]$ are all $\geqq 0$ and satisfy the inequalities (3).

Lemma C. Define $v$ and $p$ as before. Let $e \geqq 2$ and $F$ be integers; $P \geqq 0, Q \geqq 0$ and

$$
P+v p^{e k} \leqq F \leqq Q
$$

Then there exist integers $h_{1}, h_{2}, \cdots, h_{v}$, and $W$ such that 


$$
F=\sum_{\alpha=1}^{v} h_{\alpha}^{k}+p^{e} W
$$

where

$$
P p^{-e} \leqq W \leqq P p^{-e}+k Q^{(k-1) / k}
$$

Proof. It is known* that there is a solution of the congruence

$$
F \equiv \sum_{\alpha=1}^{v} h_{\alpha}^{k}\left(\bmod p^{e}\right)
$$

for $v$ and $p$ as defined. Hence there is an integer $W$ such that

$$
F=\sum_{\alpha=1}^{v} h_{\alpha}^{k}+p^{e} W
$$

For $\alpha=1,2, \cdots, v-1$ we choose $h_{\alpha}$ so that $0 \leqq h_{\alpha}<p^{e}$. Then from (4), (5) we have $P+p^{e k} \leqq F-\sum_{\alpha=1}^{v-1} h_{\alpha}^{k} \leqq Q$. In Lemma B with $m=p^{e}, E=F-\sum_{\alpha=1}^{v-1} h_{\alpha}^{k}$, one of the $p^{e}$ consecutive integers $i_{\beta}$ must be congruent to $h_{v}$ modulo $p^{e}$, and we take $h_{v}$ equal to this particular $i_{\beta}$. It follows from Lemma B that

$$
P \leqq F-\sum_{\alpha=1}^{v} h_{\alpha}^{k} \leqq P+k p^{e} Q^{(k-1) / k}
$$

and then from (5) that

$$
P p^{-e} \leqq W \leqq P p^{-e}+k Q^{(k-1) / k} .
$$

The proof of Lemma A now proceeds as follows: Let

$$
Q(\mu)=\left(2 t+2^{k}\right) p^{(k+2+\mu) k^{2}}+v p^{(k+2) k^{2}} .
$$

Since $w>p^{(k+6) k^{2}}$ and $2 t \leqq k 2^{k}$, we have

$$
\begin{aligned}
w & >p^{(k+4) k^{2}} \cdot p^{2 k^{2}}>\left(k 2^{k}+2^{k}\right) p^{(k+4) k^{2}}+4 p^{(k+2) k^{2}} \\
& \geqq\left(2 t+2^{k}\right) p^{(k+4) k^{2}}+v p^{(k+2) k^{2}}=Q(2) .
\end{aligned}
$$

Thus for every integer $w>p^{(k+6) k^{2}}$ there is an integer $\mu \geqq 2$ such that

$$
Q(\mu) \leqq w<Q(\mu+1)
$$

* See E. Landau, Vorlesungen über Zahlentheorie, vol. 1, 1927, Theorems 291 and 301 . 
Now

$$
\begin{aligned}
Q(\mu+1) & -2 t p^{(k+2+\mu) k^{2}}=\left(2 t+2^{k}\right) p^{(k+3+\mu) k^{2}}+v p^{(k+2) k} \\
& -2 t p^{(k+2+\mu) k}<\left(2 t+2^{k}\right) p^{(k+3+\mu) k^{2}} \\
\leqq & \left(k 2^{k}+2^{k}\right) p^{(k+3+\mu) k^{2}}<(2 / k)^{k /(k-1)} p^{k(k+1)(k+2)+\mu k^{2}} .
\end{aligned}
$$

Hence we have

(8)

$$
\begin{aligned}
Q(\mu)-2 t p^{(k+2+\mu) k^{2}} & \leqq w-2 t p^{(k+2+\mu) k^{2}} \\
& <Q(\mu+1)-2 t p^{(k+2+\mu) k^{2}}, \\
2{ }^{k} p^{(k+2+\mu) k^{2}}+v p^{(k+2) k^{2}} & \leqq w-2 t p^{(k+2+\mu) k^{2}} \\
& <(2 / k)^{k /(k-1)} p^{k(k+1)(k+2)+\mu k^{2}} .
\end{aligned}
$$

As in the proof of Lemma C, with $F=w-2 t p^{(k+2+\mu) k^{2}}, e=(k+2) k$, there is a solution of

$$
w-2 t p^{(k+2+\mu) k^{2}} \equiv \sum_{\alpha=1}^{v} w_{\alpha}^{k}\left(\bmod p^{(k+2) k}\right),
$$

with $0 \leqq w_{\alpha}<p^{(k+2) k}$. Hence

$$
w-2 t p^{(k+2+\mu) k^{2}}=\sum_{\alpha=1}^{v} w_{\alpha}^{k}+p^{(k+2) k} H
$$

where from (8) we have

$$
\begin{gathered}
2^{k} p^{(k+2+\mu) k^{2}} \leqq p^{(k+2) k} H<(2 / k)^{k /(k-1)} p^{k(k+1)(k+2)+\mu k^{2}}, \\
2^{k} p^{(k+2)\left(k^{2}-k\right)+\mu k^{2}} \leqq H<(2 / k)^{k /(k-1)} p^{(k+2+\mu) k^{2}} .
\end{gathered}
$$

Since

$$
2^{k} p^{(k+2)\left(k^{2}-k\right)+\mu k^{2}}>\left(2^{k}-4\right) p^{(k+2)\left(k^{2}-k\right)+\mu k^{2}}+v p^{\mu k^{2}},
$$

this gives

$$
\left(2^{k}-4\right) p^{(k+2)\left(k^{2}-k\right)+\mu k^{2}}+v p^{\mu k^{2}} \leqq H<(2 / k)^{k /(k-1)} p^{(k+2+\mu) k^{2}} .
$$

Next, by Lemma C, with $F=H, e=\mu k$,

$$
P=\left(2^{k}-4\right) p^{(k+2)\left(k^{2}-k\right)+\mu k^{2}}, \quad Q=(2 / k)^{k /(k-1)} p^{(k+2+\mu) k^{2}},
$$

there exist integers $h_{1}, h_{2}, \cdots, h_{v}$, and $W$ such that 


$$
H=\sum_{\alpha=1}^{v} h_{\alpha}^{k}+p^{\mu k} W
$$

where

$$
\begin{aligned}
\left(2^{k}-4\right) p^{(k+2+\mu)\left(k^{2}-k\right)} \leqq W \leqq & \left(2^{k}-4\right) p^{(k+2+\mu)\left(k^{2}-k\right)} \\
& +k(2 / k) p^{(k+2+\mu)\left(k^{2}-k\right)} \\
\left(2^{k}-4\right) p^{(k+2+\mu)\left(k^{2}-k\right)} \leqq W \leqq & \left(2^{k}-2\right) p^{(k+2+\mu)\left(k^{2}-k\right)}
\end{aligned}
$$

Hence from (9) and (10) we obtain

$$
w-2 t p^{(k+2+\mu) k^{2}}-\sum_{\alpha=1}^{v} w_{\alpha}^{k}-\sum_{\alpha=1}^{v}\left(p^{k+2} h_{\alpha}\right)^{k}=p^{(k+2+\mu) k^{2}} W,
$$

where $W$ satisfies the inequalities (11). This proves Lemma A.

It remains to show how the constant $C^{\prime}$ of Theorem $\mathrm{B}$ is obtained. Proceeding as in $\S 9$ of paper $\mathrm{P}$ with $s=2 k-1, s_{1}=4+\zeta_{k}$ $=s_{2}$, but using Lemma A instead of Lemma 33, we prove that $w$ is a sum of

$$
\left(2 t+s_{2}+2 v+2 k-1\right)
$$

$k$ th powers, provided that $W>C_{99}$. We shall have $W>C_{99}$ if $\mu$ is chosen so that

$$
\left(2^{k}-4\right) p^{(k+2+\mu) k^{2}}>C_{99}^{k /(k-1)} .
$$

From (6), (7) this inequality holds if

$$
w>v p^{(k+2) k^{2}}+\left(2 t+2^{k}\right)\left(2^{k}-4\right)^{-1} p^{k^{2}} C_{99}^{k /(k-1)},
$$

which proves (2).

The University of California 